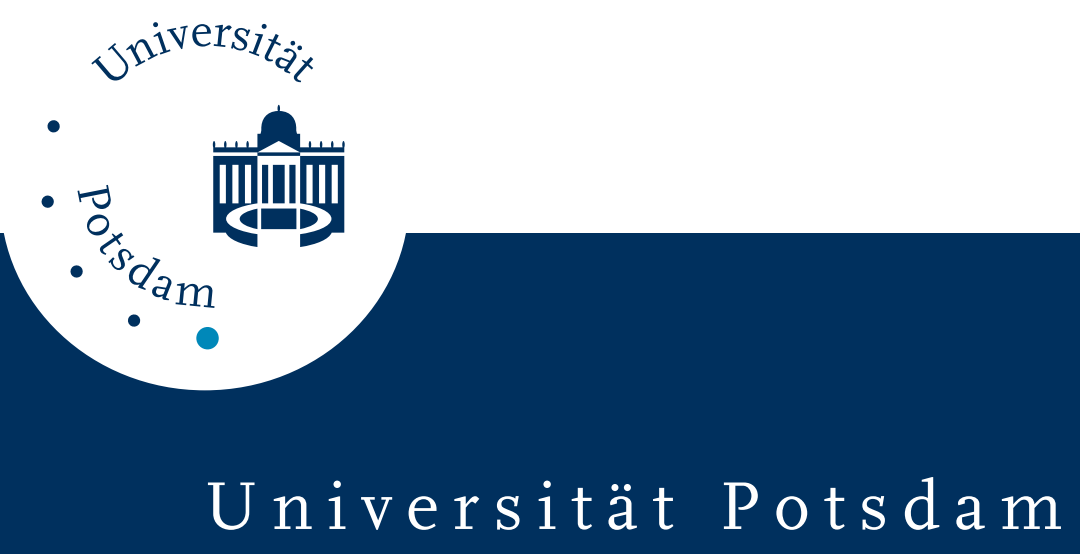

Donald Lupo, Werner Prass, Ude Scheunemann, André Laschewsky, Helmut Ringsdorf, Isabelle Ledoux

\title{
Second-harmonic generation in Langmuir-Blodgett monolayers of stilbazium salt and phenylhydrazone dyes
}

first published in:

Journal of the Optical Society of America B, 5 (1988) S. 300-307, ISSN 0740-3224

DOI 10.1364/JOSAB.5.000300

Postprint published at the Institutional Repository of Potsdam University:

In: Postprints der Universität Potsdam

Mathematisch-Naturwissenschaftliche Reihe ; 94

http://opus.kobv.de/ubp/volltexte/2009/1740/

http://nbn-resolving.de/urn:nbn:de:kobv:517-opus-17401

Postprints der Universität Potsdam

Mathematisch-Naturwissenschaftliche Reihe ; 94 


\title{
Second-harmonic generation in Langmuir-Blodgett monolayers of stilbazium salt and phenylhydrazone dyes
}

\author{
Donald Lupo, Werner Prass, and Ude Scheunemann \\ Hoechst AG, D-6230, Frankfurt, Federal Republic of Germany
}

Andre Laschewsky and Helmut Ringsdorf

Institute of Organic Chemistry, University of Mainz, D-6500, Mainz, Federal Republic of Germany

Isabelle Ledoux

Centre National d'Etudes des Télécommunications, Laboratoire de Bagneux, 196, avenue Henri Ravera, F-92220, Bagneux, France

Received August 14, 1987; accepted October 26, 1987

\begin{abstract}
The second-order nonlinear optical susceptibilities $\chi^{(2)}$ of several phenylhydrazone and stilbazium salt dyes in Langmuir-Blodgett monolayers have been determined from second-harmonic-generation measurements. Three of the substances demonstrated $\chi^{(2)}$ values greater than $10^{-6}$ electrostatic units, although two of the three did not absorb light significantly at the second-harmonic wavelength.
\end{abstract}

\section{INTRODUCTION}

The high quadratic nonlinearities of organic materials, their wide transparency range over the visible and the near-infrared spectrum, and their short response time associated with their off-resonance nonlinear properties qualify them for various applications in the field of optical signal processing, such as amplifiers, frequency converters, and modulators. An increasing number of organic materials are now shown to exhibit second-order nonlinear efficiencies that are several orders of magnitude higher than in $\mathrm{LiNbO}_{3} \cdot{ }^{1-4}$ For example, the quadratic nonlinear susceptibility $d_{21}$ of $N$-4-nitrophenyl- $L$-prolinol. (NPP) crystals is $200 \times 10^{-9}$ electrostatic units (esu), ${ }^{5}$ a value corresponding to an enhancement of 2 orders of magnitude of the nonlinear efficiency over $\mathrm{LiNbO}_{3}$.

Higher nonlinear coefficients could be obtained by using more-complex structures, such as stilbene derivatives or merocyanines. ${ }^{6,7}$ The molecular hyperpolarizabilities $\beta$ of these compounds are among the largest known for organics, but in most cases the molecular units crystallize in centrosymmetric space groups and, therefore, show no secondorder nonlinear optical effects.

The Langmuir-Blodgett (L-B) technique ${ }^{8,9}$ is one of the various methods used as a means of imposing a noncentrosymmetric structure onto a material that crystallizes in a centrosymmetric structure. L-B films for use in quadratic nonlinear optics can be ordered in a statistically noncentrosymmetric lattice.

One of the foremost features of the L-B technique is the ability to control the film thickness in the deposition process. In addition, the index of refraction can be modified, if required, for optical applications. For example, one can modify the number of $\mathrm{CH}_{2}$ groups forming the hydrophobic tail, thus simultaneously acting on the layer thickness and on the index of refraction. ${ }^{10}$ In principle, these properties qualify L-B films for giving rise to two-dimensional optical waveguides, and various studies have already been carried out in this field. ${ }^{11-13}$ However, most of the L-B films have a tendency to form microdomains in the range $2-200 \mu \mathrm{m}$ and are responsible for losses due to scattering of the optical wave propagating in the waveguide. However, it is possible, by using an appropriate thermal treatment, to obtain larger domains $(2 \mathrm{~mm})$ in a crystalline form, for example, in azobenzene molecules, ${ }^{14}$ which are good candidates for quadratic experiments.

Nonlinear-optical characterization of L-B molecules is usually achieved by measuring the second-harmonic ( $\mathrm{SH}$ ) signal emitted by the L-B film deposited upon a substrate. The measurements can be carried out either in transmission or in reflection. The SH intensity can be enhanced significantly by resonance if either or both of the fundamental and harmonic frequencies are close to an absorption band. In thick materials, the proximity of the resonance induces a strong attenuation that is not compatible with the observation of a SH signal; on the contrary, L-B films, by virtue of their thinness, do not introduce a strong attenuation of the signal and are therefore particularly suited to the observation of resonance-enhanced transverse effects. The SH signal emitted by a monolayer can therefore be detected and subsequently can be used to measure the corresponding hyperpolarizability $\beta$ and susceptibility $\chi^{(2)}$ of the deposited L-B molecule.

Second-harmonic generation (SHG) from monolayers of highly polarizable dyes was reported for the first time by Aktsipetrov et al., ${ }^{15}$ the corresponding $\chi^{(2)}$ being of the order of that of $\mathrm{LiNbO}_{3}$. SHG has been demonstrated in monolayers of merocyanine, ${ }^{16}$ hemicyanines, ${ }^{17}$ amidonitrostilbenes, ${ }^{18}$ and azobenzene derivatives. ${ }^{19} Z$-type ${ }^{19-21}$ and $Y$ type alternated multilayers ${ }^{18,22,23}$ have been studied; the corresponding $\mathrm{SH}$ signals increase with the number of active 
Table 1. Molecular Structure, Thickness Per Layer 1, Area Per Molecule, Wavelength of the Maximum Absorption Band $\lambda_{\max }$, Refractive Index $n$ at 633 $n m$, Deposition Pressure $p$, and Deposition Rate $v$ of Dye Monolayers

\begin{tabular}{|c|c|c|c|c|c|c|}
\hline $\begin{array}{c}\text { Molecular structure and } \\
\text { designation }\end{array}$ & $I(\dot{A})$ & $\begin{array}{l}\text { Area } / \\
\text { molecule }\end{array}$ & $\lambda_{\text {max }}$ & $n$ & $\left(\begin{array}{c}P \\
(m N\end{array} m^{\prime \prime}\right)$ & $\begin{array}{c}v \\
(\mathrm{~cm} / \mathrm{mn}) \\
\end{array}$ \\
\hline $\begin{array}{c}\mathrm{H}_{37} \mathrm{C}_{13}-\mathrm{O}-\mathrm{O}-\mathrm{CH}=\mathrm{CH}-\mathrm{H}^{\mathrm{N}}-\mathrm{CH}_{3} \\
1\end{array}$ & 27 & $35 \dot{A}^{2}$ & 360 & 1.6 & 25 & 1.0 \\
\hline $\begin{array}{l}\mathrm{H}_{33} \mathrm{C}_{16} \\
\mathrm{H}_{33} \mathrm{C}_{16}{ }^{\prime} \mathrm{N}\end{array}$ & 26 & $53 \dot{A}^{2}$ & 475 & 1.6 & 35 & 1.0 \\
\hline $\begin{array}{r}\mathrm{H}_{35} \mathrm{C}_{17}-\mathrm{C}-\mathrm{C} \\
0\end{array}$ & 27.5 & $24.5 \dot{A}^{2}$ & 412 & 1.57 & 35 & 1.0 \\
\hline$-\mathrm{CH}=\mathrm{N}-\mathrm{N}-\mathrm{O}-\mathrm{NO}_{2}$ & 30 & $26.5 \dot{A}^{2}$ & 420 & 1.58 & 35 & 1.0 \\
\hline $\begin{array}{c}\mathrm{H}_{33} \mathrm{C}_{15}-0-\mathrm{O}-\mathrm{CH}=\mathrm{N}-1 \\
5\end{array}$ & 27.5 & $26 \dot{A}^{2}$ & 420 & 1.61 & 35 & 1.0 \\
\hline
\end{tabular}

layers according to a subquadratic, ${ }^{20}$ quadratic, ${ }^{19}$ or superquadratic ${ }^{21}$ dependence, depending on the structure and the deposition conditions of the samples. Linear electro-optic effects of a monolayer of hemicyanine have also been demonstrated. ${ }^{24,25}$ Only a few molecules have been studied up to now, and it would be of interest to investigate new compounds that combine good film quality with large nonlinearoptical susceptibilities.

In this paper we report some $\beta$ measurements on two families of nonlinear molecules, stilbazium salt derivatives and a new class of nonlinear dyes, phenylhydrazone derivatives. The nonlinear behavior of the molecules is studied in monolayers, for various donor $\left(\mathrm{R}-\mathrm{O}-, \mathrm{R}_{2} \mathrm{~N}-, \mathrm{R}-\mathrm{CO}-\mathrm{O}-\right)$ groups associated with the same acceptor $\left(=\mathrm{HN}^{+}-\mathrm{R}\right.$ for stilbazium salts, $-\mathrm{NO}_{2}$ for the phenylhydrazone). The molecular structures of these compounds are given in Table 1.

\section{SYNTHESIS OF THE COMPOUNDS}

The synthetic routes to the compounds are shown in Fig. 1. The intermediates for the stilbazium salts as well as for the phenylhydrazones are aromatic aldehydes that are synthesized by ether formation or esterification of 4-hydroxybenzaldehyde. 4-(N,N-Dihexadecyl)-aminobenzaldehyde is prepared by Vilsmeier synthesis from the respective aniline compound.

The stilbazium salts 1 and 2 are prepared from the aldehydes and 2-methyl- $N$-methylpyridinium iodide through an aldol-condensation reaction, and the phenylhydrazones 3,4 , and $\mathbf{5}$ are obtained through condensation of the aldehydes with $p$-nitrophenylhydrazine.

\section{PREPARATION AND CHARACTERIZATION OF MONOLAYERS}

\section{A. Monolayer Isotherms}

All compounds were characterized in monolayers at the gaswater interface by isotherm measurements. ${ }^{26}$ Compound 1 exhibits only a solid analog phase in the temperature range from 20 to $40^{\circ} \mathrm{C}$. At $20^{\circ} \mathrm{C}$ the breakdown pressure range is $40 \mathrm{mN} / \mathrm{m}$, and the area required at that point is $0.28 \mathrm{~nm}^{2} /$ molecule. This large area requirement implies that the packing within the monolayer is controlled mainly by the packing of the head group and not by a tight packing of the hydrocarbon tails of the amphiphile. This tight packing of hydrocarbon chains would lead to an area requirement of approximately $0.2 \mathrm{~nm}^{2} /$ molecule for a simple chain compound.

The double-chain compound 2 exhibits a solid analog phase at $20^{\circ} \mathrm{C}$ and both solid and fluid analog phases at higher temperatures (see Fig. 2). The area at the collapse point of the monolayer is $0.4 \mathrm{~nm}^{2} /$ molecule. Thus we can assume that the monolayer packing here is controlled by a dense packing of the two hydrocarbon chains.

The spreading behavior of the phenylhydrazones is illustrated in Fig. 3 for compounds 3 and 4 . Compound 5 has almost the same isotherm as compound 4; the collapse pressure, however, is about $5 \mathrm{mN} / \mathrm{m}$ lower.

The ethers as well as the esters exhibit only solid analog phases, even at $40^{\circ} \mathrm{C}$. The collapse pressures are approximately $60 \mathrm{mN} / \mathrm{m}$ at $20^{\circ} \mathrm{C}$, and the area required at the collapse point by the ethers 4 and 5 is $0.25 \mathrm{~nm}^{2} /$ molecule, whereas the collapse point of the ester 3 is somewhat smaller $\left(0.22 \mathrm{~nm}^{2} /\right.$ molecule). Thus the hydrocarbon chains cannot be packed densely. The monolayer packing is controlled mainly by the head group. The ester can be packed better than the ether compound.

\section{B. Langmuir-Blodgett Monolayer Films}

All substances were deposited using a Lauda Langmuir trough in a clean room during the upstroke on both sides of glass microscope slides at $20^{\circ} \mathrm{C}$. The spreading and deposition conditions are described in Appendix A. The slides were cleaned and rendered hydrophilic by treatment with a 1:4 mixture of $30 \% \mathrm{H}_{2} \mathrm{O}$ and concentrated $\mathrm{H}_{2} \mathrm{SO}_{4}$ and were then rinsed with Millipore purified water.

Spectra were recorded of the monolayers and of as many 


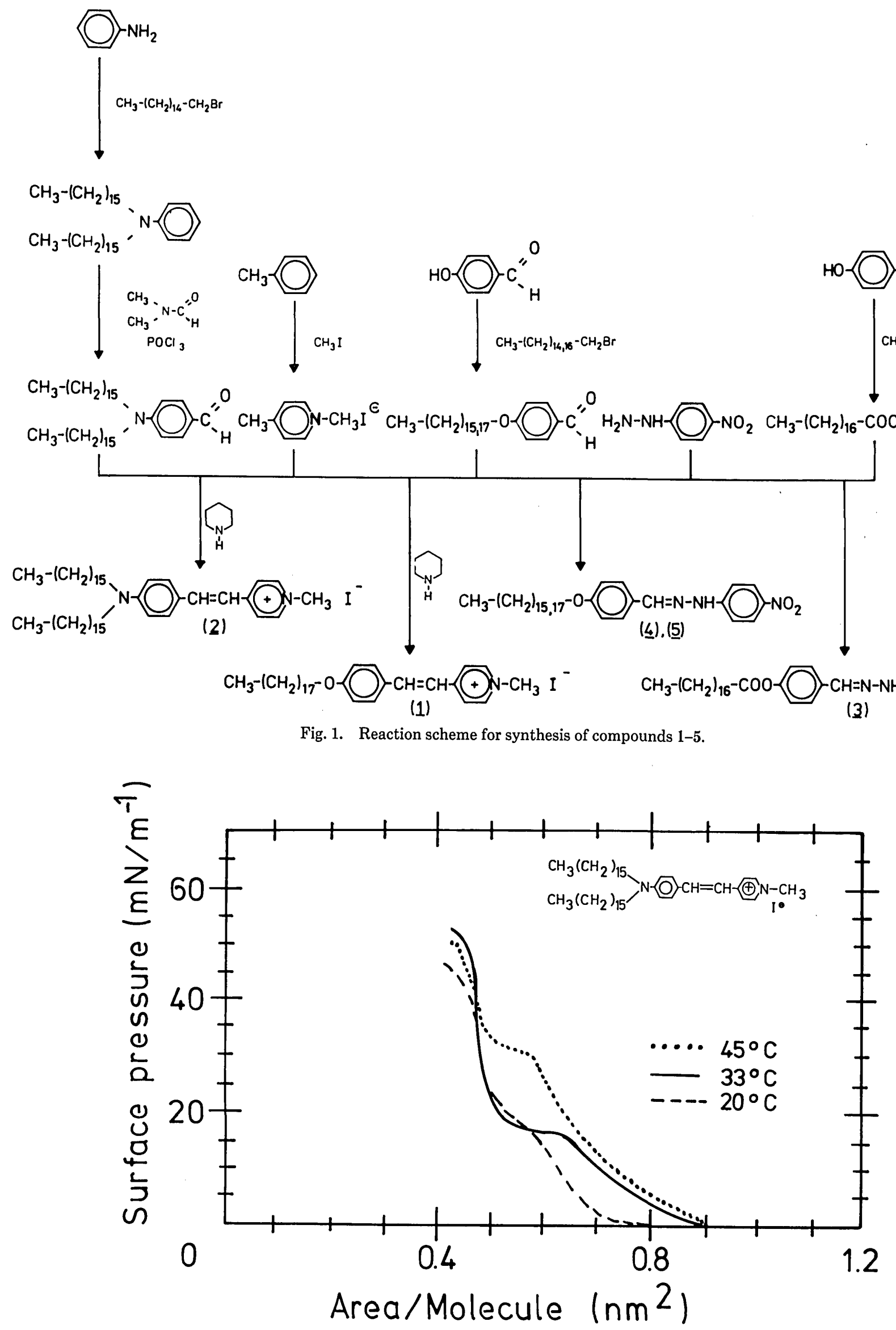

Fig. 2. Temperature dependence of the spreading behavior of compound 2 . 


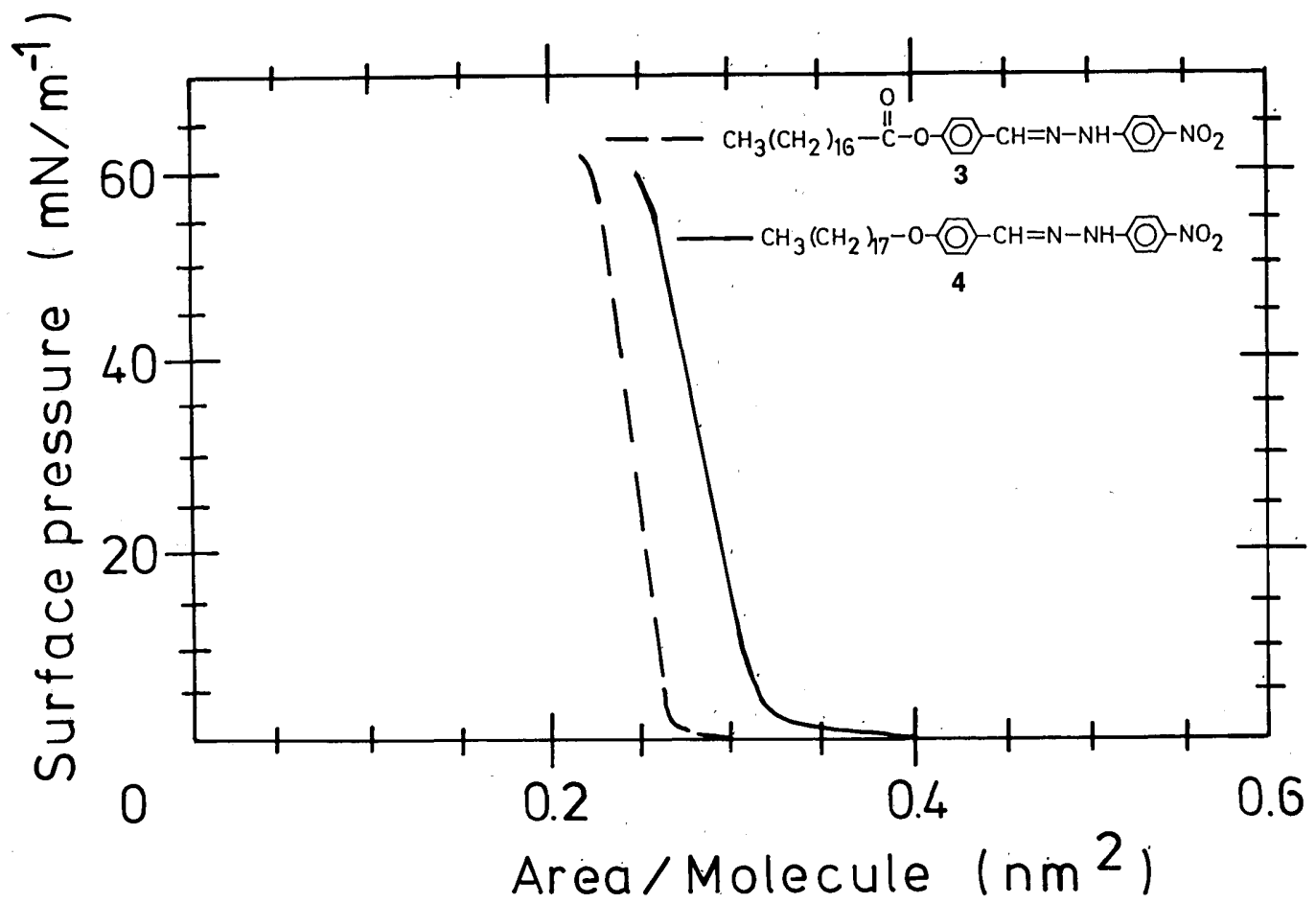

Fig. 3. Spreading behavior of compounds 3 and 4 at $20^{\circ} \mathrm{C}$.

as 40 layers of the dyes in the wavelength range $300-1000 \mathrm{~nm}$ on a Perkin-Elmer spectrophotometer. The absorption spectra of the monolayers are shown in Fig. 4.

For phenylhydrazone derivatives, the shape of the absorption peaks is very similar for the three compounds. The wavelength $\lambda_{\max }$ corresponding to the maximum of the absorption band is exactly the same for 4 and 5 , the structure of the chromophores being identical in both cases. On the contrary, the $\lambda_{\max }$ value is $115 \mathrm{~nm}$ higher for 2 than for 1 . The higher value can be accounted for by the strong electron-donor character of the disubstituted amino group in 2 compared with the poor electron-donor properties of the ether group in 1. Compound 2 has its maximum absorption wavelength close to the harmonic wavelength $\lambda=532 \mathrm{~nm}$, and a strong enhancement of $\beta$ owing to the proximity of the resonance can be expected.

The film thicknesses $l$ and refractive indices $n$ of the films at $633 \mathrm{~nm}$ were determined by performing ellipsometric measurements on as many as 30 layers on silicon substrates. Compounds 1 and 2 did not build stable multilayers; thus the refractive indices could not be determined directly. Table 1 summarizes the deposition conditions for the monolayers on glass and the results of the ellipsometric measurements. The thickness per layer $l$ is determined by measuring the thickness $t$ of $N Y$-type monolayers $(N=10,20$, or $30)$ by ellipsometric techniques, the value of $l$ being given by $l=t / N$. This value could be smaller than the length of the isolated molecule, because a partial interpenetration of the aliphatic chains belonging to two adjacent monolayers is possible. Hence, for monolayers, the relevant molecular length corresponds to the value of the isolated molecule; the measured values must therefore be corrected by considering the actual interatomic distances of each chemical bond. In fact, comparison between the experimental $l$ values measured here and the molecular lengths calculated from inter- atomic bond distances leads to similar values for phenylhydrazone derivatives; the discrepancy does not exceed $3 \%$ in any case.

On the contrary, $l$ measurements of stilbazium salts, carried out on two layers only, lead to a thickness per layer of 20 $\AA$, whereas the calculated value is $27 \AA$ for 1 and $26 \AA$ for 2 . The discrepancy occurs because the ellipsometric technique is not accurate for a very small number of $\mathrm{L}-\mathrm{B}$ layers. For the reasons mentioned above, the refractive indices for stilbazium salts could not be determined. The $n$ values for the phenylhydrazone derivatives are very similar; however, a slight increase of $n$ is observed when the length of the ali-

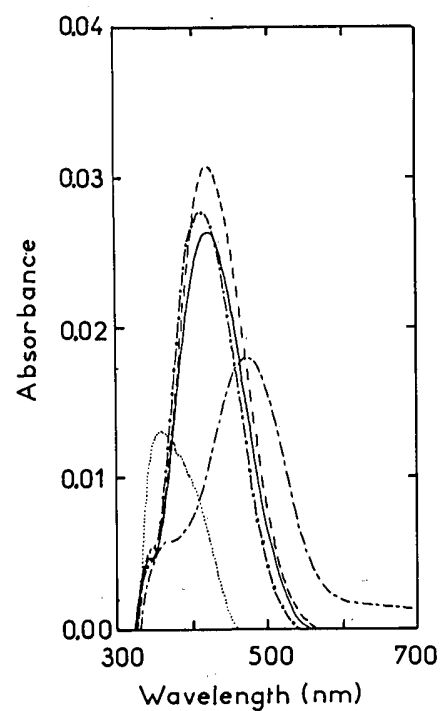

Fig. 4. Ultraviolet-visible absorption spectra of monolayers of compounds $1-5$ on glass substrates: $1, \cdots \cdots ; 2,--; 3,-\cdot-\cdot ;$ $4,-\ldots ; 5,---$ 
phatic chain is decreased, as evidenced by comparing compounds 4 and 5 , which differ only by the number of $\mathrm{CCH}_{2}+$ groups in the hydrophobic tail. This property could be used to control with good accuracy the refractive index of a L-B layer in view of applications such as mode coupling in a planar waveguide.

\section{EXPERIMENTAL SETUP FOR NONLINEAR MEASUREMENTS}

The experimental setup for the SHG measurements is illustrated in Fig. 5. The experiments were carried out using a $Q$-switched $\mathrm{Nd}^{3+}:$ YAG laser $(\lambda=1.064 \mu \mathrm{m})$. Two $\lambda / 4$ plates were used to vary the polarization direction, and the laser energy was able to be attenuated by using neutral-density filters. The fundamental beam was then focused upon the sample (focal length $60 \mathrm{~cm}$ ). The sample was set on a rotation stage monitored by a stepping motor so as to vary the incidence angle $\theta$ of the fundamental beam, the rotation axis being vertical and perpendicular to the laser beam. SHG signals were recorded at various values of $\theta$, ranging from $-50^{\circ}$ to $+50^{\circ}$. The transmitted harmonic light was detected after the remaining fundamental was filtered off by a photomultiplier and was sampled, averaged, and recorded synchronously. This detection system can measure weak harmonic signals by using a photomultiplier with a very low dark current and by improving the synchronization between the SH pulse and the trigger of the boxcar. If one works at large integration scales $(10 \mathrm{sec})$, the noise level does not exceed $0.1 \mathrm{mV}$; the minimum $\chi^{(2)}$ value of L-B films that can be detected is therefore of the order of $2 \times 10^{-9}$ esu. The measurements were calibrated against the $\mathrm{SH}$ Maker fringes emitted by a 2 -mm-thick quartz plate. The macroscopic second-order susceptibility $\chi^{(2)}$ of the L-B sample was determined relative to the $d_{11}$ coefficient of quartz $\left(d_{11}=1.2 \times\right.$ $\left.10^{-9} \mathrm{esu}\right)$. In addition, the reference used consisted of the SH signal $I_{R}{ }^{2 \omega}$ produced by a nonlinear organic powder of NPP irradiated by an additional fundamental beam and sampled and averaged by the same lock-in amplifier as the $\mathrm{SH}$ signal $I_{\mathrm{LB}}{ }^{2 \omega}$ emitted by the L-B films. The signal $I_{\mathrm{LB}}{ }^{2 \omega}$ is divided by the reference $I_{R}{ }^{2 \omega}$ and recorded. This setup considerably reduces the variations of the $\mathrm{SH}$ signal owing to laser fluctuations and therefore improves the rapidity and the sensitivity of the measurements.

The radiation emitted by the L-B films is identified as $\mathrm{SH}$

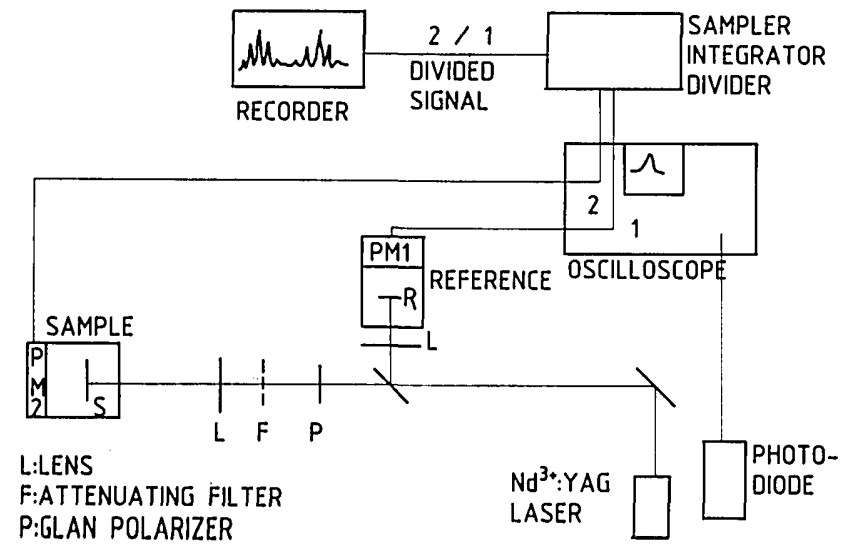

Fig. 5. Experimental setup for determination of the SHG susceptibility.

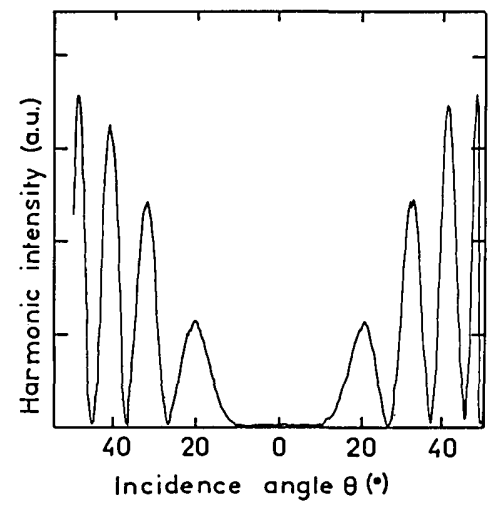

Fig. 6. Normalized SHG as a function of the incidence angle $\theta$ for 2 .

radiation by its narrow spectral width, its pulse duration shorter than that of the fundamental beam, and its quadratic dependence on incident laser energy. Regions of the samples free from the film gave no detectable signal, confirming that the observed radiation is emitted by the film only. The harmonic peak is $p$ polarized, independent of the polarization of the fundamental beam. In this work we focused only on SHG from $p$-polarized fundamental beams.

\section{NONLINEAR OPTICAL PROPERTIES}

The dependence of the emitted intensity with respect to the incidence angle shows a well-contrasted fringing pattern resulting from interference between the harmonic beams produced by the front and the back layers (Fig. 6). Because the optical path in the L-B layers (of the order of a few tens of angstroms) is much smaller than the coherence length (of the order of a few tens of micrometers), this pattern has no connection whatsoever with Maker fringes. It originates from dispersion of the refractive index of the substrate $n_{G}{ }^{2 \omega}$ - $n_{G}{ }^{\omega}$, which introduces a dephasing factor between harmonic waves generated at the front and back sides, respectively, of the substrate. ${ }^{27} n_{G}{ }^{\omega}$ and $n_{G}{ }^{2 \omega}$ are the fundamental and harmonic refractive indices of the glass substrate, respectively. The dephasing factor varies with the optical path in the substrate as the plate is rotated, as was previously demonstrated for third-harmonic generation in polydiacetylene L-B multilayers. ${ }^{27}$ The interfringe $\delta \theta$ is given by

$$
\delta \theta=\frac{\lambda}{2 L} \frac{1}{\cos \theta\left(\tan \theta_{G}{ }^{\omega}-\tan \theta_{G}{ }^{2 \omega}\right)},
$$

where $L$ is the thickness of the substrate, $\theta_{G}{ }^{\omega}$ and $\theta_{G}{ }^{2 \omega}$ are the angles of incidence of the fundamental and harmonic beams, respectively, inside the glass substrate, and $\lambda$ is the fundamental wavelength. In the pattern sketched here, the experimental interfringe between the second and the third fringes is $8.6^{\circ}$. Taking $L=1 \mathrm{~mm}, n_{G} \omega=1.50806$, and $n_{G}{ }^{2 \omega}=$ $1.52083, \delta \theta=8.7^{\circ}$. This result confirms the origin of this $\mathrm{SH}$ fringe pattern resulting from the dispersion of the refractive index of the substrate.

From the envelope function of the $\mathrm{SH}$ fringe pattern we can infer both the $\chi^{(2)}$ value of the sample and the average tilt angle $\bar{\phi}$ between the charge-transfer axis of the molecules and the normal to the substrate. $\chi^{(2)}$ and $\bar{\phi}$ are adjusted to give the best fit between the theoretical and the experimen- 
tal envelope function of the fringes. The second-harmonic intensity from the L-B sample is given by

$$
\begin{aligned}
I_{\mathrm{LB}}{ }^{2 \omega}= & \frac{K^{\mathrm{LB}} l}{\lambda^{2}} F\left(\theta, \theta_{F}{ }^{\omega, 2 \omega}, \theta_{G}{ }^{\omega, 2 \omega}, n_{F}{ }^{\omega, 2 \omega}, n_{G}{ }^{\omega, 2 \omega}, \bar{\phi}\right) \\
& \times\left[\chi^{(2)}\right]^{2}\left(E^{\omega}\right)^{4} \cos \delta Q,
\end{aligned}
$$

where the function $F$ includes factors related to the transmittance and reflectance of the various interfaces as a function of angles of incidence in the film for fundamental or harmonic beams $\theta_{F}{ }^{\omega}$ or $\theta_{F}^{2 \omega}$, respectively, and in the glass substrate $\theta_{G}{ }^{\omega}$ or $\theta_{G}{ }^{2 \omega}$. $F$ depends also on the refractive index at fundamental (harmonic) frequencies in the film $n_{F}{ }^{\omega}\left(n_{F}{ }^{2 \omega}\right)$ and in glass $n_{G}{ }^{\omega}\left(n_{G}{ }^{2 \omega}\right)$ and on the angle $\theta . \quad \chi^{(2)}$ is the nonlinear coefficient of the L-B film of thickness $l$, the projection factors depending on the tilt angle $\bar{\phi}$ being taken into account in the envelope function $F . \delta Q$, describing the phase difference between the two harmonic beams, has the form

$$
\delta Q=\frac{2 \omega L}{c}\left(n_{G}{ }^{2 \omega} \cos \theta_{G}{ }^{2 \omega}-n_{G}{ }^{\omega} \cos \theta_{G}{ }^{\omega}\right) .
$$

$K^{\mathrm{LB}}$ is a factor independent of the orientation of the sample, including the absorption coefficient of the film at $\omega$ and $2 \omega$. $\chi^{(2)}$ values are determined by comparison of the amplitude of the envelope of the interference fringes with the signal from the quartz reference plate. This calibration eliminates the term $\left(E^{\omega}\right)^{4}$, where $E^{\omega}$ is the amplitude of the incident fundamental field, which cannot be measured directly when currently available $\mathrm{Nd}^{3+}$ :YAG laser cavities are used.

The molecular hyperpolarizabilities are deduced from the $\chi^{(2)}$ values by using a molecular-oriented gas model in which

$$
\chi^{(2)}=\tilde{N} f^{2 \omega}\left(f^{\omega}\right)^{2} \beta .
$$

Here $f^{\omega, 2 \omega}=\left[\left(n^{\omega, 2 \omega}\right)^{2}+2\right] / 3$ is a local field factor and $\tilde{N}$ is the number of molecules per unit volume, equal to $\sigma / l$, where $\sigma$ is the surface density of the monolayer. Therefore

$$
\beta=\frac{\chi^{(2)} l}{f^{2 \omega}\left(f^{\omega}\right)^{2} \sigma} .
$$

Table 2. $\chi^{(2)}, \beta$, and Tilt Angle $\bar{\phi}$ for Dye Monolayers

\begin{tabular}{|c|c|c|c|c|}
\hline Molecule & $\bar{\phi}(0)$ & $\chi^{(2)}(\times 10 \%$ esu $)$ & $\beta\left(\times 10^{27} /\right.$ esu $)$ & Ref. \\
\hline 1 & 50 & 0.51 & 0.15 & $\begin{array}{c}\text { This } \\
\text { work }\end{array}$ \\
\hline 2 & 50 & 6.25 & 2.0 & $\prime \prime$ \\
\hline 3 & 65 & 0.56 & 0.11 & $\prime \prime$ \\
\hline 4 & 60 & 1.80 & 0.40 & $\prime \prime$ \\
\hline 5 & 55 & 1.70 & 0.33 & $\prime \prime$ \\
\hline Merocyanine & 9 & - & 4.06 & 16 \\
\hline Hemicyanine & 43 & 0.95 & 0.31 & 17 \\
\hline D P N A & 65 & 1.60 & 0.31 & 20 \\
\hline
\end{tabular}

[The validity of the Lorenz approximation involved in Eq. (4) is not strictly verified in the case of monomolecular layers placed at the glass-air interface. This model would be more nearly valid for thick multimolecular L-B samples. Therefore the $\beta$ value given by Eq. (5) corresponds to the hyperpolarizability of a monolayer that would be surrounded by L-B layers of the same nature rather than to the $\beta$ value of a monolayer placed at the glass-air interface.]

$\bar{\phi}, \chi^{(2)}$, and $\beta$ values for the five molecules studied here are given in Table 2. It is of interest to compare these values with nonlinear data measured in other L-B molecules, such as merocyanines and hemicyanines or diazostilbene derivatives, and to examine the influence of some electron-donor or electron-attractor substituents on the nonlinear properties of L-B molecules. The relative errors on $\chi^{(2)}$ and $\beta$ do not exceed $10 \%$.

\section{DISCUSSION}

In each of the two families reported here, the acceptor group remains the same, and the donor group is modified. Therefore the electron-donor character of these substituents can be compared. Of the phenylhydrazone derivatives, 4 and 5 have similar $\beta$ values when the experimental errors are taken into account; the hyperpolarizability is slightly stronger for the highest chain length (4). This small increase of $\beta$ with respect to the chain length is probably due to a slight inductive effect of the hydrophobic tail, the electron-donor character of a linear alkyl substituent $\mathrm{R}=\mathrm{C}_{n} \mathrm{H}_{2 n+1}$ increasing with the number of carbons $n$. Comparison between 3 and 4 or 3 and 5 indicates that the ester substituent $-\mathrm{H}_{35} \mathrm{C}_{17}-\mathrm{COO}-$ is a very poor electron-donor group, the corresponding $\beta$ value being much smaller than for the other $\mathrm{L}-\mathrm{B}$ molecules reported up to now for quasi-identical resonance effects (the wavelength of the absorption maximum being almost the same for 3 and for 4 or 5). The results obtained on the two stilbazium salts confirm the strong electron-donor character of the disubstituted amino group $\left(\mathrm{H}_{33} \mathrm{C}_{16}\right)_{2} \mathrm{~N}$ - when compared with the $\mathrm{H}_{37} \mathrm{C}_{8}-\mathrm{O}-$ group of compound 1 . In this last case, the small $\beta$ value can be accounted for by the weak electron-donor character of the ether substituent and by the weaker resonance effect between the harmonic frequency and the first absorption peak of the molecule, which lies at $360 \mathrm{~nm}$; both phenomena have a cumulative influence on the weakening of $\beta$. On the other hand, the electron-donor power of the $(\mathrm{R})_{2} \mathrm{~N}$ - group in 2 is reinforced by the presence of two long aliphatic chains grafted onto the nitrogen atom. In addition, the presence of two hydrophobic tails on a L-B molecule instead of one should increase the stability and therefore the ordering of the monolayer. But the most important feature is the proximity of the maximum absorption wavelength $\lambda_{\max }$ to the harmonic frequency, leading to a large enhancement of the nonlinearity. In fact, the $\chi^{(2)}$ value is high (more than 2 orders of magnitude higher than for $\mathrm{LiNbO}_{3}$ ), and the hyperpolarizability is at least six times higher than for the hemicyanine reported in Ref. 17. In fact, 2 exhibits one of the highest quadratic nonlinearities ever measured, its $\beta$ value coming just behind that of the merocyanine described in Ref. 16 . Indeed the value for $\chi^{(2)}$ of 6.5 $\times 10^{-6}$ esu is the largest explicitly reported volume susceptibility known $\left[\chi^{(2)}\right.$ was not given for the merocyanine]. Another factor that could be responsible for this 
exceptionally large hyperpolarizability is the nature of the substituent grafted onto the pyridinium ion: the long aliphatic chain used in Ref. 6 has a higher electron-donor character than the $\mathrm{CH}_{3}$ group grafted onto 2 and therefore has a tendency to reduce the electron-attractor power of the pyridinium ion. In principle, this effect is not strong, but previous $\beta$ measurements, carried out by electric-field-induced SHG (EFISHG) in solution, on $N$-octadecylmerocyanine (NOM), ${ }^{6}$ and on $N$-methylmerocyanine (NMC), ${ }^{7}$ led to similar conclusions: the $\beta$ value is 5-10 times smaller for NOM than for NMC, although the fundamental wavelength used for $\operatorname{NOM}(\lambda=1.318 \mu \mathrm{m})$ is closer to the resonance than the wavelength used for NMC $(\lambda=1.89 \mu \mathrm{m})$. (The solvent was dimethylsulfoxide for NMC and methanol or pyridine for NOM.) Although such results must be considered carefully, the EFISHG measurements seem to confirm this influence of the chain length on the electron-attractor character of the pyridinium ion. The highest $\beta$ value known currently is that of the merocyanine indicated in Table 2 . This molecule can be described by the two mesomeric structures:

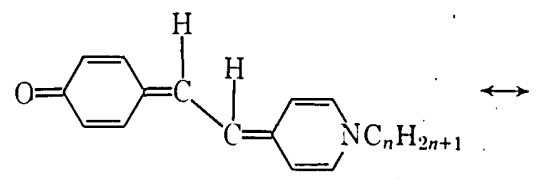

(A)

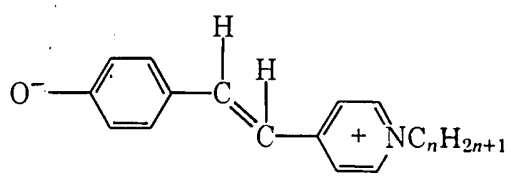

(B)

The (B) form corresponds to a conjugated system into which has been substituted the strongest electron-donor group $-\mathrm{O}^{-}$and the strongest electron-acceptor group pyridinium ion. The large charge transfer resulting from this structure is responsible for its high $\beta$ value.

Therefore these measurements have confirmed the classification of various chemical groups following an increasing electron-donor character:<smiles>[R]OC(=O)C([R])O[13CH]N(C)C</smiles>

and an increasing electron-acceptor character:

$$
-\mathrm{NO}_{2}<\mathrm{N}^{+}-\mathrm{C}_{n} \mathrm{H}_{2 n+1}<\mathrm{N}^{+}-\mathrm{CH}_{3}
$$

\section{CONCLUSION}

We have measured $\chi^{(2)}$ and $\beta$ values of various nonlinear molecules and illustrated some molecular-engineering rules for the electron-donor and the electron-acceptor character of some substituents. The coupling of the best donor and acceptor groups led to an exceptionally nonlinear L-B molecule, with a $\beta$ value of $2 \times 10^{-27}$ esu. SHG measurements on L-B monolayer's of nonlinear molecules are convenient means for determining the $\beta$ values of various structures.
The highly efficient compound reported here seems to be promising for further investigations on multilayers and for applications in forming planar waveguides for quadratic nonlinear interactions. Some studies on highly efficient multilayers are currently in progress.

\section{APPENDIX A}

\section{A. Synthesis of Compounds}

\section{Compound 1}

$5.8 \mathrm{~g}$ ( $42 \mathrm{mmol}$ ) of dry $\mathrm{K}_{2} \mathrm{CO}_{3}$ is suspended in a solution of 4.9 $\mathrm{g}(40 \mathrm{mmol})$ of 4-hydroxybenzaldehyde, $13.3 \mathrm{~g}(40 \mathrm{mmol})$ of 1 -bromooctadecane, and $50 \mathrm{mg}(0.3 \mathrm{mmol})$ of $\mathrm{KI}$ in $300 \mathrm{ml}$ of dry acetone. ${ }^{28}$ The suspension is refluxed for $48 \mathrm{~h}$ and filtered when still hot. $300 \mathrm{ml}$ of hexane is added, and the solution is washed with $10 \% \mathrm{Na}_{2} \mathrm{CO}_{3}$ in water. After the residual water is removed with $\mathrm{Na}_{2} \mathrm{SO}_{4}$, the solvent is evaporated under reduced pressure, and the crude reaction product is recrystallized from hexane (melting point, $74^{\circ} \mathrm{C}$; yield, $80 \%)$.

$1.1 \mathrm{~g}(2.9 \mathrm{mmol})$ of 4-octadecyloxybenzaldehyde, $1.7 \mathrm{~g}$ (3 $\mathrm{mmol}$ ) of $\mathrm{N}$-methyl-4-methylpyridinium iodide, and $1 \mathrm{ml}$ of piperidine are dissolved in $50 \mathrm{ml}$ of ethanol and refluxed for $5 \mathrm{~h}$. After it is cooled to room temperature, the reaction mixture is filtered. The precipitate is collected, dissolved in chloroform, and washed with $1 \mathrm{M} \mathrm{HI}$ and water. After evaporation of the solvent in vacuo the product is recrystallized from hexane and methanol [melting point, $>200^{\circ} \mathrm{C}$ (decomposed); yield, 86\%].

\section{Compound 2}

$10 \mathrm{~g}$ (18.5 mmol) of $N, N$-dihexadecylaniline is dissolved in $20 \mathrm{~g}$ (270 mmol) of freshly distilled dimethylformamide. ${ }^{29,30}$ The solution is cooled down to $5^{\circ} \mathrm{C}$, and $2.8 \mathrm{~g}$ (18.3 mmol) of $\mathrm{POCl}_{3}$ is added within $5 \mathrm{~min}$. The mixture is stirred for $1 \mathrm{~h}$ at $20^{\circ} \mathrm{C}$ and then for $3 \mathrm{~h}$ at $80^{\circ} \mathrm{C}$. After it is cooled down again, the mixture is hydrolyzed by slowly adding $40 \mathrm{~g}$ of icecold water and is then neutralized with approximately $10 \mathrm{ml}$ of $5 \mathrm{M} \mathrm{NaOH}$. The precipitate is collected and recrystallized from methanol, hexane, and ethanol (melting point, $57^{\circ} \mathrm{C}$; yield, $48 \%$ ).

$1.7 \mathrm{~g}(3 \mathrm{mmol})$ of 4 -( $N, N$-dihexadecyl)-aminobenzaldehyde, $0.7 \mathrm{~g}(3 \mathrm{mmol})$ of $N$-methyl-4-methylpyridinium iodide and $1 \mathrm{ml}$ of piperidine are dissolved in $50 \mathrm{ml}$ of ethanol and refluxed for $3 \mathrm{~h}$. After the mixture is cooled to room temperature, the precipitate is collected, dissolved in chloroform, and washed with $1 \mathrm{M} \mathrm{HI}$ and water. After evaporation of the solvent the product is recrystallized several times from ethanol [melting point, $>200^{\circ} \mathrm{C}$ (decomposed); yield, $81 \%]$.

\section{Compound 3}

$6.11 \mathrm{~g}(50 \mathrm{mmol})$ of 4-hydroxybenzaldehyde and $8 \mathrm{ml}$ of triethylamine are dissolved in $200 \mathrm{ml}$ of absolute methylene chloride. ${ }^{28}$ The solution is cooled down to $5^{\circ} \mathrm{C}$, and a solution of $15.1 \mathrm{~g}(50 \mathrm{mmol})$ of stearic acid chloride in $100 \mathrm{ml}$ of absolute methylene chloride is added within 20 min. After being heated to room temperature, the reaction mixture is washed with $1 \mathrm{M} \mathrm{HCl}$, water, $5 \% \mathrm{Na}_{2} \mathrm{CO}_{3}$, and again with water. After removal of residual water with $\mathrm{Na}_{2} \mathrm{SO}_{4}$ the 
solvent is evaporated in vacuo. The crude reaction product is recrystallized twice from methanol (melting point, $60^{\circ} \mathrm{C}$; yield, $66 \%)$.

$3.89 \mathrm{~g}(10 \mathrm{mmol})$ of the stearic acid ester of 4-hydroxybenzaldehyde is added to a solution of $1.53 \mathrm{~g}(10 \mathrm{mmol})$ of 4 nitrophenylhydrazine in a mixture of $10 \mathrm{ml}$ of glacial acetic acid, $10 \mathrm{ml}$ of water, and $100 \mathrm{ml}$ of ethanol. The mixture is stirred for $2 \mathrm{~h}$ at room temperature, and the precipitate is collected and recrystallized repeatedly from methanol, ethyl acetate, and acetone (melting point, $134^{\circ} \mathrm{C}$; yield, $24 \%$ ).

\section{Compound 4}

$1.2 \mathrm{~g}$ ( $7.8 \mathrm{mmol})$ of 4-nitrophenylhydrazine is dissolved in a mixture of $10 \mathrm{ml}$ of glacial acetic acid, $10 \mathrm{ml}$ of water, and 100 $\mathrm{ml}$ of ethanol. ${ }^{28}$ To that $2.7 \mathrm{~g}(9.7 \mathrm{mmol})$ of octadecyloxybenzaldehyde is added, and the mixture is stirred for $2 \mathrm{~h}$ at room temperature. The precipitate is collected and recrystallized repeatedly from hexane and methanol (melting point, $112^{\circ} \mathrm{C}$; yield, $68 \%$ ).

\section{Compound 5}

Compound $\mathbf{5}$ is synthesized according to the same procedure as for compound 4, starting from $3.47 \mathrm{~g}(10 \mathrm{mmol})$ of 4 hexadecyloxybenzaldehyde and $1.53 \mathrm{~g}(10 \mathrm{mmol})$ of 4-nitrophenylhydrazine (melting point, $117^{\circ} \mathrm{C}$; yield, $66 \%$ ).

\section{B. Film-Balance Measurements}

The water used for all film-balance experiments was purified with a Millipore water purification system (Milli-Q, 4 bowl). This system was fed with deionized water.

Chloroform was used as solvent for spreading compounds 1 and 2, and methylene chloride was used for compounds 3, 4, and 5. All solvents were purchased from Merck (Uvasol). The concentration of the spreading solutions was $0.5 \mathrm{mg} / \mathrm{ml}$.

The isotherms were measured with a commercial computer-controlled film balance (Filmwaage 2, MGW Lauda) with a sweep time of $10 \mathrm{~min} /$ curve. The same film balance in combination with the corresponding film lift was used for the deposition experiments.

The cleanliness of the film balance and the purity of the water were checked routinely by measuring isotherms of a well-known compound (palmitic acid, reference for GC). ${ }^{31}$ The purity of the spreading solvents was also checked by this method.

\section{ACKNOWLEDGMENTS}

It is a pleasure to thank D. Josse and P. Fremaux for setting up the laser source and the detection system, P. Frank for film preparation, and P. Ottenbreit for synthetic work. We are much indebted to J. Zyss for fruitful discussions.

\section{REFERENCES}

1. D. J. Williams, ed., Nonlinear Optical Properties of Organic and Polymeric Materials, ACS Symposium Series 233 (American Chemical Society, Washington, D.C., 1983).

2. G. Khanarian, ed., Molecular and Polymeric Optoelectronic Materials, Proc. Soc. Photo-Opt. Instrum. Eng. 682 (1986).

3. D. S. Chemla and J. Zyss, eds., Nonlinear Optical Properties of Organic Molecules and Crystals (Academic, New York, 1987).

4. J. B. Stamatoff, A. Buckley, G. Calundann, E. W. Choe, R.
DeMartino, G. Khanarian, T. Leslie, G. Nelson, D. Stuetz, C. C. Teng, and H. Y. Yoon, "Development of polymeric nonlinear optical materials," in Molecular and Polymeric Optoelectronic Materials, G. Khanarian, ed., Proc. Soc. Photo-Opt. Instrum. Eng. 682, 85-92 (1986).

5. I. Ledoux, D. Josse, P. Vidakovic, and J. Zyss, "Highly efficient single-crystalline organic thin films for quadratic nonlinear optics," Opt. Eng. 25, 202-210 (1986).

6. B. F. Levine, C. G. Bethea, E. Wasserman, and L. Leenders, "Solvent dependent hyperpolarizability of merocyanine dye," J. Chem. Phys. 68, 5042-5045 (1978).

7. A. Dulcic and C. Flytzanis, "A new class of conjugated molecules with large second-order polarizability," Opt. Commun. 25, 402406 (1978).

8. I. Langmuir, "The constitution and fundamental properties of solids and liquids. II. Liquids," J. Am. Chem. Soc. 57, 18481906 (1917).

9. K. B. Blodgett, "Films built by depositing successive monomolecular layers on a solid surface," J. Am. Chem. Soc. 57, 10071022 (1935).

10. J. Zyss, "Nonlinear organic materials for integrated optics: a review," J. Molec. Electron. 1, 25-45 (1985).

11. G. Stegeman, "Nonlinear integrated optics," J. Appl. Phys. 58, R57-R78 (1985).

12. W. L. Barnes and J. R. Sambles, "Guided optical waves in Langmuir-Blodgett films of 22-tricosenoic acid," Surf. Sci. 177, 399-416 (1986).

13. A. J. Vickers, R. H. Tredgold, P. Hodge, E. Khoshdel, and I. Girling, "An investigation of liquid-crystal side-chain polymeric Langmuir-Blodgett films as optical waveguides," Thin Solid Films 132, 43-48 (1985).

14. R. Jones, R. H. Tredgold, A. Hoorfar, R. A. Allen, and P. Hodge, "Crystal formation and growth in Langmuir-Blodgett multilayers of azobenzene derivatives: optical and structural studies," Thin Solid Films 132, 57-66 (1985).

15. O. A. Aktsipetrov, N. N. Akhmediev, E. D. Mishina, and V. R. Novak, "SHG on reflection from a monomolecular Langmuir layer," JETP Lett. 37, 207-209 (1983).

16. I. R. Girling, P. V. Kolinsky, and C. M. Montgomery, "Observation of SHG from a Langmuir-Blodgett monolayer of a merocyanine dye," Electron. Lett. 21, 169-170 (1983).

17. I. R. Girling, N. A. Cade, P. V. Kolinsky, R. J. Jones, I. R. Peterson, M. M. Ahmad, D. B. Neal, M. C. Petty, G. G. Roberts, and W. J. Feast, "SHG in mixed hemicyanine: fatty-acid Langmuir-Blodgett monolayers," J. Opt. Soc. Am. B 4, 950-955 (1987).

18. D. B. Neal, M. C. Petty, G. G. Rober, G. G. Ahmad, W. J. Feast, I. R. Girling, N. N. Cade, P. V. Kolinsky, and I. R. Peterson, "SHG from Langmuir-Blodgett superlattices containing two active components," Electron. Lett. 22, 460-462 (1986).

19. O. A. Aktsipetrov, N. N. Akhmediev, I. M. Baranova, E. D. Mishina, and V. R. Novak, "Structure of Langmuir-Blodgett films by second-harmonic reflection," Sov. Phys. JETP 62, 524530 (1985).

20. I. Ledoux, D. Josse, P. V. Vidakovic, J. Zyss, R. A. Hann, P. F. Gordon, B. D. Bothwell, S. K. Gupta, S. Allen, P. Robin, E. Chastaing, and J.-C. Dubois, "SHG by Langmuir-Blodgett multilayers of an organic azo dye," Europhys. Lett. 3, 803-809 (1987).

21. L. M. Hayden, S. T. Kowel, and M. P. Srinivasan, "Enhanced SHG from multilayered Langmuir-Blodgett films of dye," Opt. Commun. 61, 351-356 (1987).

22. I. R. Girling, P. V. Kolinsky, N. A. Cade, J. D. Earls, and I. R. Peterson, "SHG from alternating Langmuir-Blodgett films," Opt. Commun. 55, 289-292 (1985).

23. I. R. Girling, N. A. Cade, P. V. Kolinsky, J. D. Earls, G. H. Cross, and I. R. Peterson, "Observation of SHG from Langmuir-Blodgett multilayers of a hemicyanine dye," Thin Solid Films 132, 101-112 (1985).

24. G. H. Cross, I. R. Girling, I. R. Peterson, and N. A. Cade, "Linear Pockels response of a monolayer Langmuir-Blodgett film," Electron. Lett. 22, 1111-1113 (1986).

25. G. H. Cross, I. R. Girling, I. R. Peterson, N. A. Cade, and J. D. Earls, "Optically nonlinear Langmuir-Blodgett films: linear 
electro-optic properties of monolayers," J. Opt. Soc. Am. B 4, 962-968 (1987).

26. G. L. Gaines, Insoluble Monolayers at the Gas-Water Interface (Interscience, New York, 1966).

27. F. Kajzar, J. Messier, J. Zyss, and I. Ledoux, "Nonlinear interferometry in Langmuir-Blodgett multilayers of polydiacetylene," Opt. Commun. 45, 133-137 (1983).

28. A. P. Phillips, "Condensation of aromatic aldehydes with $\gamma$-picoline methiodide," J. Org. Chem. 14, 302-305 (1949).
29. Organikum (VEB Verlag der Wissenschaften, Berlin, 1977), p. 409.

30. Organikum (VEB Verlag der Wissenschaften, Berlin, 1977), p 484.

31. O. Albrecht, "The construction of a microprocessor-controlled film balance for precision measurement of isotherms and isobars," Thin Solid Films 99, 227-234 (1983). 